\title{
Akademischer Austausch Schweiz-Deutschland: 40 Jahre Wechselspiel
}

Ernst G. Jung

Korrespondenz:

Prof. Dr. med. Ernst G. Jung

Maulbeerweg 20

D-69120 Heidelberg

ernst.g.jung[at]t-online.de
Zum 1. Oktober 1965 fuhren wir, Prof. Urs Walter Schnyder, der dem Ruf auf den Lehrstuhl «Dermatologie und Venerologie» an die Universität Heidelberg folgte, und ich als sein leitender Oberarzt, von Zürich nach Heidelberg. Wir fuhren über Stuttgart, um uns beim Schweizerischen Generalkonsul persönlich vorzustellen. Die Familien mit jeweils drei Kindern folgten mit dem Hausrat einige Wochen später. So waren wir ausgewandert.

Empfangen wurden wir in Heidelberg an Klinik und Universität, in der Stadt und im privaten Umfeld mit gespannten Erwartungen, sehr herzlich und mit «Vorschusslorbeeren». Wir trafen auf eine offene, freundliche Einstellung, die wir so nicht erwarteten, und wurden angenehm überrascht, ja stimuliert. Unser Anfang gelang, und ich konnte mich 1968 für unser Fach Dermatologie an der Uni Heidelberg habilitieren.

\section{Fieberjahre der deutschen Universitäten (Epoche der 68er Jahre)}

Da aber waren wir schon mitten in den Fieberjahren der deutschen Universitäten, in Unruhen und Umwälzungen, die weit in die allgemeine Politik und die sozialen Strukturen sich «einbissen» und als «Die 68er Jahre» epochalen Charakter erlangten [1]. Die Schweizer Dozenten in Deutschland wurden keineswegs verschont, zumal mehrere in exponierten Funktionen hervortraten. Vier deutsche Universitäten hatten Schweizer Rektoren:

- Freiburg: Prof. Hansjürg Steinlin (1921-2004), Forstwirt, Rektor 1970-73, Präsident WRK 1977-80

- Mainz: Prof. Peter Schneider (1920-2002), Jurist, Rektor 1969-74, Präsident der Uni 1974-80

- Frankfurt: Prof. Walter Rüegg, (1918), Soziologe, Rektor 1965-70, Präsident WRK 1967-68

- Heidelberg: Prof. Kurt Baldinger (1919-2007), Romanist, Rektor 1968-69

Sie wirkten auch massgeblich mit in der Westdeutschen Rektorenkonferenz (WRK) und versuchten, Rationalität und Neuerungen zu verbinden unter Aufrechterhaltung einer leistungsorientierten Universität. Ein hehres Unterfangen, eine SisyphusArbeit, die letztlich scheitern musste. Kurt Baldinger hat damals mit resignativem Unterton von der «Beresina-Situation» gesprochen, anspielend auf die aufopfernde Verteidigung der letzten Beresina- brücke durch die Schweizer Bataillone beim Rückzug der «Grande Armée» Napoleons aus Russland 1813.

Zudem wurde ein gewisser «Schulterschluss» gesucht. In den späten 60er und frühen 70er Jahren gab es eine jährlich zusammengerufene, informelle Versammlung der Schweizer Hochschuldozenten in der BRD, eingeführt von Prof. Walter Rüegg mit der Schweizer Botschaft in Bonn und den Generalkonsulaten Frankfurt und Stuttgart sowie getragen von Schweizer Firmen in der BRD: Nestlé in Frankfurt a. M., ABB in Mannheim und Wild-Leitz in Giessen.

Die Gastfreundschaft der Schweizer Unternehmungen in der BRD sowie die Anlehnung an die diplomatischen Vertretungen waren sehr wertvoll und «stärkten den Rücken».

Beabsichtigt war ein Zusammenschluss der Dozenten mit Informationsaustausch angesichts der überhandnehmenden Unruhen und politischen Agitationen an den Universitäten sowie auch der Störungen der Lehre und Verunglimpfungen der Forschung. Auch die Verbindung zur Akademischen Schweiz sollte gefestigt werden. Auf administrativer Ebene durch die Mitwirkung des Leiters der Auslandschweizer-Organisation in Bern, Herrn Ney, und akademisch durch Gastreferenten wie Prof. Gerhard Huber, Philosoph und Präsident des Schweizerischen Wissenschaftsrates, Prof. Ernst Hadorn, Zoologe und Entwicklungsbiologe aus Zürich und, ebenfalls Zoologe, der philosophische Anthropologe Prof. Adolf Portmann aus Basel.

Es waren damals ca. 170 Schweizer Dozenten in der BRD tätig und, nach Aussage des Botschafters, eine annähernd gleich grosse Zahl deutsche Dozenten in der Schweiz. Also ein paritätischer Austausch, wenn man die beiden Länder als Einheiten betrachtet und den Grössenunterschied ausser Acht lässt.

Betrachtet man den Unterschied an Fläche und Bewohnern, so muss man die «Lieferantenseite», die Geber, unterscheiden von der «Empfängerseite». Denn die Schweiz hatte damals ca. 6 Millionen Einwohner, die BRD wohl 63 Millionen, also 10-mal mehr. Die Schweiz lieferte in die BRD 10-mal mehr Dozenten pro eigene Bevölkerung (also 1 Dozent pro $35000 \mathrm{CH}$-Einwohner), als umgekehrt. Nur 1 Dozent auf 370000 Bewohner der BRD wirkte in der Schweiz. Ein markanter «Brain Drain» aus der Schweiz in die BRD also; von der Schweiz aus gesehen, in die sog. «Diaspora». 
Drei vordergründige Argumente waren:

- Personell: Ergänzung der vom Krieg mehr als dezimierten Jahrgänge in Forschung und Lehre;

- Inhaltlich: Nacharbeitung der kriegsbedingt fehlenden Kontinuität der Forschung (oder der nur einseitigen Entwicklung);

- Geistig: Hilfe bei der Erarbeitung demokratischer Denk- und Organisationsformen, auch in der Lehre.

Etwas hintergründig mochte man gelegentlich auch von einem nicht unwillkommenen «Abfluss» eines Überschusses qualifizierter Hochschullehrer der Schweiz sprechen. Tatsache ist, dass die Schweiz damals ihren Bedarf an Hochschullehrern leicht und qualifiziert aus dem eigenen Nachwuchs decken konnte.

Der Aspekt des Empfängers aber sah schon damals um 1970 anders aus. Zunächst wurde der virtuelle Verlust von ca. 170 Schweizer Hochschullehrern, die dauernd in der BRD waren, durch eine vergleichbare Zahl deutscher Hochschullehrer in der Schweiz ausgeglichen, wie auch umgekehrt. Allerdings machten die deutschen Hochschullehrer in der Schweiz in der Gesamtheit aller Hochschullehrer der Schweiz einen wesentlich mächtigeren Anteil aus als umgekehrt. Der Unterschied betrug 1:10, ist beeindruckend und wurde, oft subjektiv beladen, auch registriert. Zuweilen wurde sogar vor einer tendenziellen «Verfremdung» der Schweizer Hochschullandschaft gewarnt.

\section{Wie ging es weiter?}

Seit den 80er Jahren haben solche Treffen der Schweizer Hochschullehrer in Deutschland nicht mehr stattgefunden, sie waren offenbar nicht mehr nötig, der hochschulpolitische Bedarf hatte sich erledigt. Die Wissenschaftskulturen haben sich angeglichen und die Forschungsleistungen ebenfalls. Der Austausch an Forschern und Wissenschaftlern hin und her wurde freier und leichter. Die Verbindung der Schweizer Hochschullehrer in der BRD zur Akademischen Schweiz verlor aber einen wichtigen und die Identität erhaltenden Anker. Dieser fehlte besonders den Medizinern, da die Schweizerische Ärztorganisation FMH ihre Auslandssektion auflöste und die Kollegen im Ausland den kantonalen Sektionen zuwies. Ein Versuch, dies zu heilen, verlief 2011 erfolglos.

Der «Brain Drain» schien sich ausgeglichen und ausgewogen zu gestalten, bis in den letzten Jahren in der Schweiz, mit Schwerpunkt Zürich, eine bemerkenswerte und als störend empfundene Vielzahl an deutschsprachigen Hochschullehrern aus Deutschland moniert und bemängelt wird. Die auffallende Dichte an Deutschen in leitenden Positionen in der Schweiz wird auch in anderen Berufsfeldern und in den nichtstaatlichen Unternehmungen angemahnt. Emotionale Auswüchse liessen sich nicht vermeiden und riefen die Politik auf den Plan. Sorge bereitet eine mögliche Bedrohung des eigenen, schweizerischen Nachwuchs bei der Besetzung leitender Positionen, insbesondere an den Hochschulen (Uni ZH und ETH) und manch einer wähnte eine Verdrängung des heimischen Schweizerdeutsch als Umgangssprache durch Hochdeutsch, so wie es in den global agierenden Bereichen Wissenschaft und Handel durch die «Weltsprache» Englisch weitgehend schon geschehen ist. Ein mächtiger «Brain Drain» aus Deutschland in die Schweiz wird moniert und aktives Gegensteuern verlangt.

In dieser manchmal auch angeheizten Debatte geht ganz vergessen, dass es immer noch und in beträchtlicher Zahl aktive und erfolgreiche Schweizer Hochschullehrer an deutschen Universitäten und Forschungseinrichtungen gibt, die gut, nahtlos und wirkungskräftig integriert sind. In vielen Fächern findet, entsprechend den internationalen Gepflogenheiten, ein reger und reibungsarmer gegenseitiger Austausch statt.

\section{Dazu Trends und einige Zahlen}

In den vergangenen 40 Jahren hat sich die Hochschullandschaft in der Schweiz und in Deutschland gewaltig gewandelt, entwickelt und differenziert. Neue Bildungsanstalten sind entstanden. Die Vielfalt der Studiengänge ist frappierend, die Zielorientierung ausgezeichnet und die neuen Benennungen herausfordernd. Die Strukturen der Forschenden und Lehrenden sind vielfältiger geworden, so dass Vergleiche schwer zu werten sind. Tatsache ist ein gewaltiger Mehrbedarf an Hochschullehrern in der Schweiz und in Deutschland, der anhand der Professoren in den Diskussionen mit Zahlen belegt wird. Eine Vermehrung um das 4-Fache ist unbestritten. Und rein zahlenmässig sind es immer noch annähend gleich viele Professoren, die gegenseitig ausgetauscht werden, eher sogar etwas mehr Schweizer in Deutschland als umgekehrt [2]. Und die absolute Zahl deutscher Professoren in der Schweiz nimmt weiter zu. Dazu einige Zahlen aus der Debatte 2013 [2, 3].

\begin{tabular}{|c|c|c|}
\hline & $\begin{array}{l}\text { Schweizer Professoren } \\
\text { in der BRD }\end{array}$ & $\begin{array}{l}\text { Deutsche Professoren } \\
\text { in der } \mathrm{CH}\end{array}$ \\
\hline 1970 & са. 170 & са. 170 \\
\hline 2008 & 772 & - \\
\hline 2009 & - & 719 \\
\hline 2012 & - & 796 \\
\hline
\end{tabular}

Die deutschen stellen unter den ausländischen Professoren in der Schweiz die weitaus grösste Gruppe dar. Die Zahlen [2-5] der drei Schwerpunkte sind:

\begin{tabular}{lllll} 
& & \multicolumn{2}{c}{$\begin{array}{l}\text { Anzahl } \\
\text { Professoren }\end{array}$} & \multicolumn{2}{c}{ Davon Deutsche } \\
Uni Zürich & 2009 & 496 & 173 & $35 \%$ \\
& 2013 & 531 & 193 & $36 \%$ \\
\hline ETH ZH & 2009 & 388 & 120 & $31 \%$ \\
\hline Uni Bern & 2009 & 352 & 115 & $33 \%$
\end{tabular}




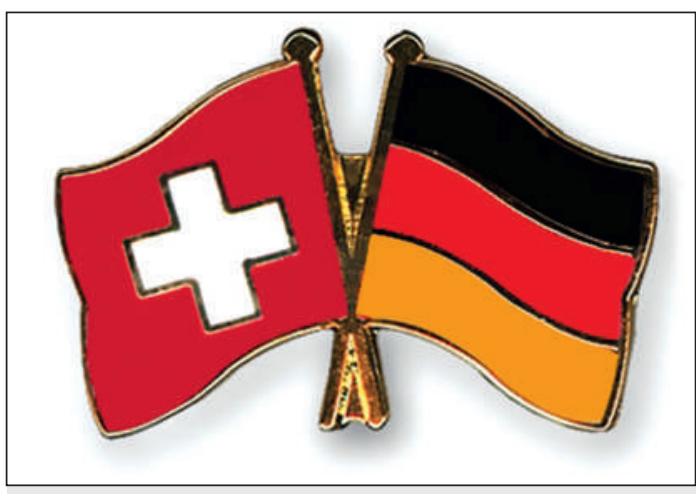

Entspricht einer notwendigen und akademisch fruchtbaren Gepflogenheit: der rege Austausch von Professoren zwischen Deutschland und der Schweiz.

Zusammenfassend kann festgestellt werden, dass ein reger Austausch von forschenden und lehrenden Professoren zwischen Deutschland und der Schweiz einer notwendigen und akademisch fruchtbaren Gepflogenheit entspricht. Rein zahlenmässig ist der Austausch vor 40 Jahren und heute annähernd gleich und hat sich in dieser Zeitspanne in beiden Richtungen vervierfacht. Absolut betrachtet ist der «Brain Drain» in beiden Richtungen also gleich mächtig und wirksam. Betrachtet man aber den «Brain Drain» in seiner Auswirkung auf den Personalbestand der «Empfänger-Hochschulen», also die deutschen Professoren in Relation zu deren Schweizer Kollegen, so ist es an den Schwerpunkten Zürich und Bern evident, dass deren Lehrkörper zu einem Drittel aus deutschen Kollegen besteht. Eine bemerkenswerte Zahl, die verständlicherweise hinterfragt wird, zu Emotionen anstachelt und Gefühle des Unbehagens verursacht. Nicht die Qualität der Lehre und Forschung wird angezweifelt, doch die allgemeine Akzeptanz der deutschen Kollegen ist gefährdet und beeinträchtigt. Emotionen und Ängste auf beiden Seiten sind die Folgen.

Ganz anders werden die Schweizer Hochschullehrer in Deutschland gut und problemlos akzeptiert und integriert, obschon sie, hinter Österreich, die grösste Gruppe ausländischer Hochschullehrer in Deutschland bilden.

Und ein weiterer Unterschied bestand und besteht noch. Während die Schweizer Hochschullehrer in Deutschland in der Zeit akademischer und politischer Unruhen um 1970 herum eine informelle Vereinigung zum «Schulterschluss» etablierten mit Hilfe der Industrie und unterstützt durch unsere diplomatischen Repräsentanten, fehlte damals den deutschen Dozenten in der Schweiz jegliche Struktur und Hilfe. Dies hat sich auch gewandelt. Nun verfügen die deutschen Hochschullehrer in der Schweiz im «Swiss German Club» über eine gut strukturierte Organisation, die vor Ort und fachspezifisch Beratung und Rückendeckung anbietet. Uns aber, den Schweizer Professoren in Deutschland, fehlt eine solche. Dabei fehlt weniger die Integrationshilfe, son- dern die Verbindung zur akademischen Schweiz und eine entsprechende Rückendeckung.

Hier kommt nun die Schweizerische Akademie der Medizinischen Wissenschaften (SAMW) ins Spiel.

\section{Wünsche an die SAMW}

Die SAMW verfügt in ihrem Senat über eine Kategorie «Korrespondierende Mitglieder». Sie kann aussergewöhnliche Gelehrte im Ausland dazu berufen und hat damit die Möglichkeit, die im Ausland wirkenden Schweizer Hochschullehrer sozusagen locker mit der akademischen Schweiz zu verbinden und auch zu verpflichten.

Gegenwärtig hat die SAMW (seit 1992) 21 korrespondierende Mitglieder, Schweizer Wissenschaftler, die dauernd im Ausland tätig sind.

10 in USA, 9 in Deutschland (4 davon in Heidelberg) und 2 in Österreich. Auffallend ist, dass bisher keine Kollegen aus Frankreich und Italien dabei sind, zeigt doch die Präsenz an den Jahressitzungen der SAMW, dass die Kollegen aus den Nachbarländern besonderes Interesse zeigen und die Verbindung zu schätzen wissen. Eine sprachliche Schwierigkeit besteht nicht, ist doch Französisch und Italienisch an den Sitzungen der SAMW allzeit präsent und allen ist Englisch geläufig.

Dieses Instrument der korrespondierenden Mitglieder möge erhalten und gestärkt werden und auch jüngere Schweizer Kollegen erfassen. Dies zumal die neuen Statuten 2013 erlauben, solches Bemühen auch auf hervorragende, nicht-medizinische Gelehrte auszudehnen, die sich in engem Bezug zur medizinischen Forschung in der Schweiz auszeichnen.

Die SAMW ist mit den anderen vier Akademien der Schweiz zusammengefasst $\mathrm{zu}$ «Akademien der Wissenschaften Schweiz». Dabei fällt auf, dass neben der SAMW nur noch eine der anderen Akademien, die SATW, das Instrument der «Korrespondierenden Mitgliedschaft» kennt.

Es ist zu wünschen, dass die SAMW im Bereich der Medizin, und die «Akademie Schweiz» für alle akademischen Bereiche das Instrument der "Korrespondierenden Mitgliedschaft» erhöhen und ausweiten, um so den Schweizer Hochschullehrern im Ausland eine Anbindung an die Akademische Schweiz und den wünschenswerten Rückhalt daselbst zu bieten.

Wir wären dankbar!

\section{Literatur}

1 Gassert, Philipp. Das kurze «1968» zwischen Geschichtswissenschaft und Erinnerungskultur. Neuere Forschungen zur Protestgeschichte der 68er Jahre. Online auf «H-Soz-u-Kult» Vom 30.4.2010.

2 Swissinfo.ch vom 2.12.2013.

3 Statistisches Lexikon der Schweiz 2012

4 NZZ. Internat. Ausgabe 21 vom 27.1.2014.

5 Statistisches Bundesamt Deutschland, zit. nach [2]. 